American Journal of Infectious Diseases 3 (4): 240-247, 2007

ISSN 1553-6203

(C) 2007 Science Publications

\title{
HIV Medication Adherence and Substance Use: The Smartest Women's Project
}

\author{
${ }^{1}$ Eliot Lopez, ${ }^{2}$ Deborah L Jones, ${ }^{1}$ Mary Ishii, ${ }^{3}$ Jonathan N Tobin and ${ }^{1}$ Stephen M Weiss \\ ${ }^{1}$ University of Miami Miller School of Medicine, ${ }^{2}$ Barry University, ${ }^{3}$ Clinical Directors Network
}

\begin{abstract}
Antiretroviral (ARV) medication for substance users has been a controversial issue with respect to whether current substance users can successfully maintain their medication regimens. This study compared ARV adherence across current substance users, former substance users and those with no history or current use and the relative impact of a medication adherence intervention on all three groups. Of the 481 predominantly African American and Latina women from Miami, New York and New Jersey enrolled in the SMARTEST Women's Program, 338 participants were prescribed antiretroviral medication at study entry. All three groups, current users $(n=60)$, former users $(n=107)$ and never users $(n=171)$, reported relatively high levels of adherence at baseline. Of those participants with less than $80 \%$ adherence at baseline, former users showed the most significant decrease in viral load post-intervention and at long term (two year) follow-up. These findings suggest former users to be the most reliable source of self-reported adherence and to profit most from the study intervention. They also suggest that additional research on targeted interventions for current substance users may be necessary to improve medication adherence for this group of women living with HIV.
\end{abstract}

Key words: Adherence, antiretroviral therapy, women, substance use, psychosocial

\section{INTRODUCTION}

Adherence to HIV treatment regimens is critical to optimal disease management, yet rates of adherence to antiretroviral (ARV) medications are frequently less than ideal and negatively affect long-term goals for disease management, including drug resistance, poor health outcomes and treatment failure ${ }^{[1,2]}$.

Adherence to medication regimens has been associated with clinically significant viral load reductions ${ }^{[3]}$. Most patients $(81 \%)$ have complete viral suppression at greater than $95 \%$ adherence, compared with increasingly less (64\%) at $90-95 \%$ adherence and only $50 \%$ at $80-90 \%$ adherence ${ }^{[2]}$. Resistance to ARV therapy is the most significant limiting factor in the long-term efficacy of HIV pharmacotherapy and it is a predictable consequence of suboptimal adherence to medication regimens ${ }^{[4-6]}$.

While controlled clinical trials of ARV therapy noted above have demonstrated viral suppression below detectable limits when adherence reaches $95 \%$ or greater, adherence rates in clinical settings rarely surpass $50 \%^{[7,8]}$.

Substance users represent an especially problematic population regarding medication prescription and adherence. Studies consistently link substance use to low levels of medication adherence ${ }^{[9-}$ ${ }^{112]}$. Because of the critical relationship between adherence and effectiveness of medication (including the development of viral resistance to entire classes of medications), medical practitioners have been reluctant to prescribe medication if there was any indication that the individual would have less than optimal adherence $^{[13]}$.

This study of HIV seropositive women living in an urban context sought to assess the influence of current or former substance use as well as different types of substance use on HIV medication adherence. The study also examined the role of various psychological factors associated with medication adherence and offers recommendations for treatment with this challenging population.

\section{MATERIALS AND METHODS}

Study design: Women were recruited from the SMART/EST Women's Project II, an NIMH-supported multisite intervention study seeking to improve the health status and quality of life of culturally diverse women living with HIV. Beginning in 2000, participants were recruited from the three major epicenters in the United States for seropositive women:

Corresponding Author: $\quad$ Stephen M Weiss, Ph.D., MPH, Department of Psychiatry \& Behavioral Sciences, University of Miami Miller School of Medicine, 1400 NW $10^{\text {th }}$ Ave, Miami, Florida 33136, USA 
Florida's Miami-Dade County, New York City and the New Jersey metropolitan area. Candidates were drawn from hospital outpatient clinics, community health centers/agencies and participant referrals. Eligible participants were 338 HIV positive women, 18 years or older, who were prescribed antiretroviral medications at study entry. Participants were requested to provide their primary care physician's approval for study participation.

The parent study included several health promotion components relevant to improving participants' health status, including nutrition, physical activity, sexual risk and substance use reduction previously cited ${ }^{[14]}$. All participants also participated in the medication adherence component, emphasizing the importance of high levels of medication adherence (i.e., > 95\%), the phenomenon of medication resistance, problems of maintaining optimal levels of adherence (e.g., forgetting, too busy, side effects), addressing these issues in a problem-solving format.

Exclusion criteria were limited to active psychosis, psychotic depression, or current substance dependence. Of the 387 persons on ARVs consented, 49 persons (13\%) were temporarily or permanently excluded. Temporary exclusion was rescinded when candidates presented evidence of being enrolled in treatment. Only 10 persons of the 387 candidates were permanently excluded based upon their "run-in" status (failure to appear for two consecutive scheduled screening appointments without notifying study personnel).

Assessments: Assessments were conducted by trained interviewers and were collected at baseline, post-intervention and at long-term follow-up (24 months post baseline). These assessments included:

* Demographic information: age, religion, nationality, ethnicity, educational level, employment status, residential status, marital status/current partner status, living situation, number of children and serostatus, date of HIV diagnosis.

* Substance use: Participants were identified as current users (those persons reporting use of controlled substances within the last 7 days); or using alcohol more than 3 drinks per day on average over 7 days ( 21 drinks a week or more), former users, e.g., having a history of substance use (history of attending AA or NA meetings or enrollment in a drug/alcohol rehabilitation program with no substance use in the last 7 days), or never users, e.g., no history of substance use (not meeting the criteria for substance use or history outlined in this paragraph). Among current users, we identified users of specific types of substances and compared substance use practices. Persons considered substance-dependent were temporarily excluded until they provided evidence of being enrolled in a substance abuse treatment program. Consistent with the literature, none of the participants in the "current user" group identified as being injecting drug users.

* Medication adherence: Adherence to anti-retroviral medication was measured by 4-day self-report using the ACTG (AIDS Clinical Trials Group) Questionnaire for Adherence to Anti-HIV Medications (4 days ${ }^{[15]}$ ). The mean number of pills per day was divided by the prescribed number using information regarding the medication regimen provided by the participant, to calculate an average adherence percentage. Frequency of medication-related experiences was measured using a Likert scale of never (0), rarely (1), sometimes (2) and often (3).

* d) Depression: Depression was assessed by the Beck Depression Inventory (BDI ${ }^{[16,17]}$ ). The newest revision of the BDI, which takes into account insomnia vs. hypersomnia and weight gain versus weight loss, was utilized. Scores were obtained for both cognitive and somatic symptoms of depression, given the likelihood of HIV seropositive patients reporting somatic symptoms which might be indicative of HIV medication side effects or depression.

* Medication beliefs: The Beliefs about Medicines Questionnaire $\left(\mathrm{BMQ}^{[18]}\right)$ was used to assess participants' beliefs about antiretroviral therapy (ART); two five-item scales assessing beliefs regarding the necessity of taking medication (Cronbach's alpha $=.80$ ) and concerns (Cronbach's alpha $=.82)$ about its potential negative consequences. Participants were asked to rate their level of agreement with statements about medication using a Likert-like scale ( $5=$ strongly agree to 1 = strongly disagree). "Concerns" assessed actual experiences with medication as well as abstract beliefs about potential future problems. Scores were summed to constitute scale scores and means obtained to create a range of 1 5 for three scales: beliefs about medications in general (GEN), beliefs about the necessity of ART (NEC) and concerns about the side effects of ART (CRN).

* Coping /social support: Coping strategies and social support were measured by a revised version of the Brief $\mathrm{COPE}^{[19]}$, a 38-item version of the COPE specifically tailored to persons living with HIV/AIDS. For the purposes of the present study, 
the behavioral disengagement subscale, which targets alcohol and drug use, was utilized. Additionally the emotional support subscale provided information on the degree of social support experienced by the participant. Items are rated from 1 (I haven't been doing this at all) to 4 (I've been doing this a lot). Alpha reliabilities for the Brief COPE exceeded minimum standards in the validation sample $(\alpha=.80)$. Possible scores ranged from 2 to 8 for each subscale, with higher scores indicating greater use of the strategy.

* Self efficacy: Self-efficacy is defined as an individual's belief in their ability to restructure distress-provoking thoughts ${ }^{[20,21]}$. The measurement of self efficacy utilizes Beck's model of cognitive therapy ${ }^{[22]}$. The Self-Efficacy Inventory is a seven item questionnaire designed to evaluate participants' perceived ability to respond to the challenges of HIV/AIDS, utilize cognitive behavioral strategies and to adhere to antiretroviral treatment. Each participant was asked to rate her confidence level to perform the skill on a 5-point Likert-type scale with polar anchors labeled "not at all" and "all of the time."

* Viral load: Viral Load was assessed from blood samples collected at baseline and all subsequent measurement timepoints using sterile evacuated tubes containing EDTA. The viral load was estimated via reverse transcriptase polymerase chain reaction using the Roche (Nutley NJ) Amplicor HIV Monitor Ultrasensitive Assay Kit and the Biomerieux (Marcy l'Etoile, France) NucliSens HIV-1 QT assay kit as described in Barre-Sinoussi et al. ${ }^{[23]}$ and Kievits et al. ${ }^{[24]}$ ). Because of the variability in viral load data, the values were log-adjusted, with subsequent analyses conducted using the adjusted values rather than raw data values.

Participants: Of those enrolled in the SMART/EST Women's Program II $(\mathrm{n}=481), 338$ participants $(78 \%)$ were prescribed antiretroviral therapy (ART) at study entry. The following descriptions relate only to those 338 participants. Participant's mean age was $42 \pm 9.13$; the largest proportion (44\%) were African-American, 24\% Puerto Rican, 13\% Haitian, 2\% Cuban, 1\% Columbian, 5\% Central American and 3\% White nonHispanic. Most (77\%) were unemployed, 10\% were working part time and 3\% full-time. The primary routes of HIV infection were sexual contact (71\%) and drug use $(6 \%)$, with $22 \%$ being uncertain of the cause of their infection. The majority $(56 \%)$ of participants reported having completed at least a $10^{\text {th }}$ grade high school education; a large proportion had histories of drug $(39 \%)$ and/or alcohol dependence (15\%). Over half of study participants $(56 \%)$ had incomes below the poverty line. It is important to note that none of those reporting injecting drug use in the parent sample $(\mathrm{n}=481)$ were prescribed ART at study entry. These data are corroborated by the significantly higher log viral loads observed among heroin users in the total sample (Fig. 1).

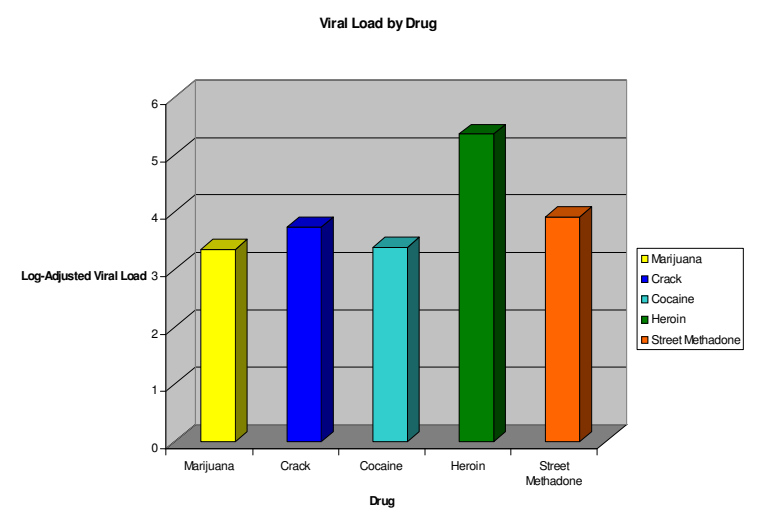

Fig. 1: Baseline viral load by type of substance use

Statistical analyses: This study used a repeated measures design with substance use (current, previous, none) as the between-subjects factor and time (baseline, post-intervention and long term followup) as the within-subjects factor. Correlations are reported as Pearson's r statistics; repeated measures between arms are reported as F statistics and effects of time on the sample are reported as t tests; all comparisons used an alpha (2-tailed) of 0.05. Data was analyzed using the Statistical Package for Social Sciences (SPSS).

Baseline analyses are based on those participants who reported being currently prescribed ART $(n=338)$ at baseline. Follow-up analyses are based on those participants who reported being currently prescribed ART and for whom a complete data set was collected, including baseline, post-intervention and long-term follow-up [ $n=228$; current users $(n=42)$, former users $(n=70)$, never users $(n=116)]$. Participant retention rates at post-intervention were $72 \%$ and long-term (24 months) follow-up were $67 \%$; attrition was reported by participants as being due primarily to illness, change of residence, employment and scheduling difficulties.

\section{RESULTS}

\section{Baseline analyses:}

Adherence: Of those currently receiving ART, 31\% (n $=105$ ) reported a history of substance abuse (former 
users), $18 \%(n=64)$ were current users and $51 \%(n=$ 169) were never users. Self-reported adherence did not differ between these three groups $(t[1,337]=.054, p=$ .96). At baseline, never users had adherence rates of $93 \%$ (SD = 19.7), former users had adherence rates of $95 \%(\mathrm{SD}=13)$ and current users reported adherence rates of $91 \%(\mathrm{SD}=19)$. In addition, there were no differences between types of medications prescribed to former users, never users and current users $(F[2,225]$ $=1.972, p=.141)$. Measures of adherence among current users suggest specific drug use is associated with accuracy of self report as measured by viral load (presented in Fig. 2).

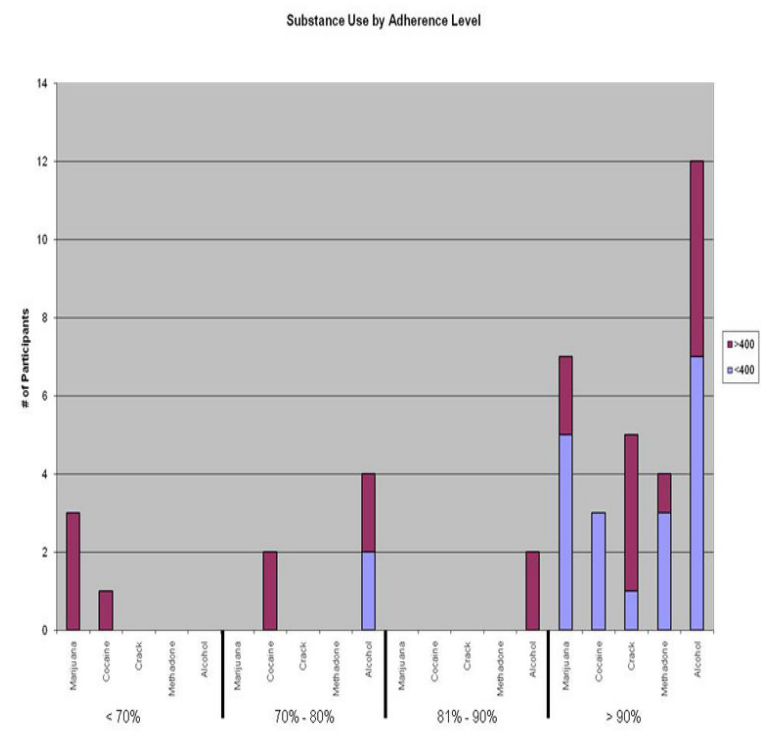

Fig. 2: Baseline adherence level by current substance use

For those women who were adherent $<80 \%$ of the time, never users had adherence rates of $51.5 \%$, former users had adherence rates of $61.5 \%$ and current users reported adherence rates of $58.5 \%$.

Adherence level was associated $(\mathrm{r}=.137, \mathrm{p}=.013)$ with nucleoside reverse transcriptase inhibitor (NRTI) usage for the entire sample. There was no difference in adherence between non-nucleoside reverse transcriptase inhibitors (NNRTIs), protease inhibitors (PIs), combination therapies (comprising a combination of two or more NRTIs, NNRTIs, PIs) and other medications under investigation. Among never users, adherence was associated with NRTI usage $(r=.198, \mathrm{p}$ $=.01$; increased adherence was associated with NRTI prescription). However, there was a negative relationship between adherence and PI usage for the same group $(\mathrm{r}=-.16, \mathrm{p}=.04$; less PI prescription was associated with higher adherence). Among both former users and current users, no relationships were found between adherence and the prescription of a particular medication.

Depression: No differences in overall levels of depression were observed between former users and never users $(t[1,337]=.125, p=.90)$. However, cognitive measures of depression differed between the two groups. Former users were an average of 1.55 points higher in symptoms of cognitive depression than never users $(t[1,337]=-2.688, p<.01)$. There were no differences in depression scores between current users and the other two groups.

Beliefs and concerns: There was no association between perceived necessity of medication, concerns regarding medication and overall levels of adherence $(t$ $[1,337]=.006, p=.92, t[1,337]=-.061, \mathrm{p}=28, t$ $[1,337]=-.028, p=.62)$. Beliefs about the necessity of medications in general were associated with concerns about HIV medications $(\mathrm{r}=.398, \mathrm{p}<.001)$. No significant differences in either beliefs or concerns were found between the three groups.

Coping/Social Support: Level of adherence was associated with Emotional Social Support (ESS; $\mathrm{r}=$ $.118, \mathrm{p}=.033$ ). Those women adherent more than $80 \%$ of the time had higher levels of Emotional Social Support $(t[1,324]=-2.789, p=.01)$, with a mean ESS score 1.54 points higher than non-adherent women. Among the three groups, former users had higher levels of social support than both never users $(t$ [254.85] $=$ $2.85, p<.01)$ and current users $(t[162]=2.083, p=$ .039). No differences were found in coping or ESS between never users and current users.

Self-efficacy: There was no association between selfefficacy and adherence rates $(\mathrm{r}=.08, \mathrm{p}=.17)$. A significant difference in self-efficacy was found amongst the three groups $(F[2,225]=5, p=.007)$. Post-hoc analysis revealed that former users had greater levels of self-efficacy than never users. Interestingly, current users had greater levels of self-efficacy than never users, as well.

Viral load: Viral load data supported the observation that former users reported higher and more accurate levels of adherence, but differences between groups were not significant. Figure 3 illustrates the relative viral load by level of adherence. 


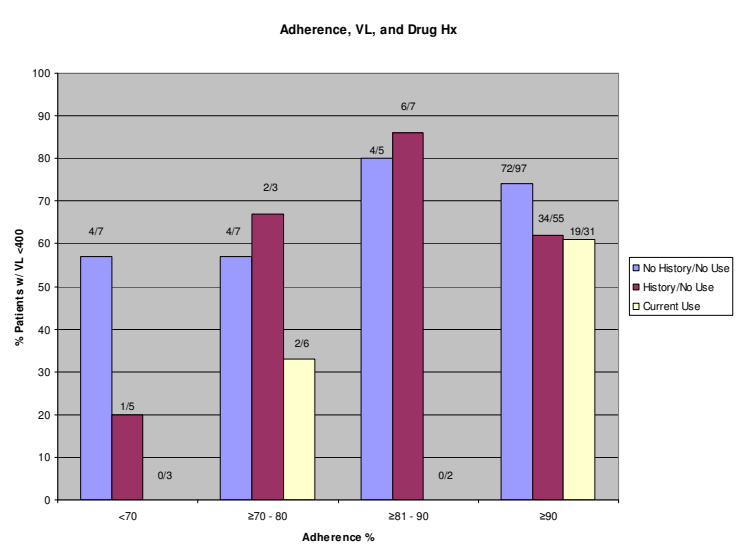

Fig. 3: Baseline viral load by adherence and drug use history

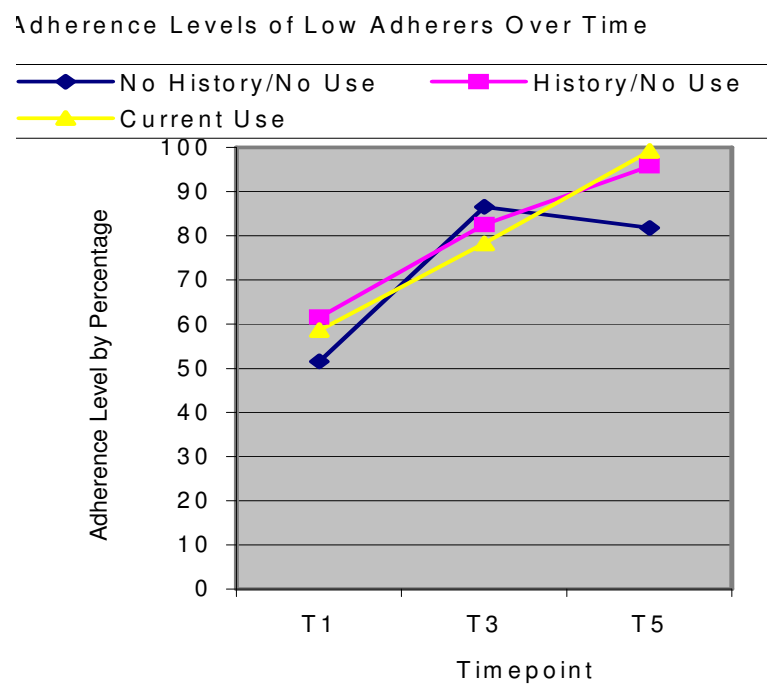

Fig. 4: Changes in adherence among low adherers over time

\section{Long term follow-up analyses:}

Adherence: There was a significant increase in self reported adherence rates at long-term (two year) follow-up for participants who reported baseline adherence levels of less than $80 \%$ (low adherers; $t[1$, $42]=-8.377, p<.001)$. Significant increases in adherence were found for former users $(t[9]=-5.17, p$ $=.001)$, current users $(t[10]=-5.478, p<.01)$ and never users $(t[21]=-5, p<.01)$. For never users whose initial adherence rates were less than $80 \%$, there was a $30 \%$ increase in reported adherence, resulting in a change in adherence rates from $52 \%$ to $82 \%$. Former users reported an adherence rate of $95 \%$. Current users achieved an adherence rate of $99 \%$ (Fig. 4). However, when self-reported adherence was matched against log viral load changes, only the former users appeared to show the expected inverse relationship between self report and biological outcomes (log viral load; Fig. 5). Viral Load Over Time

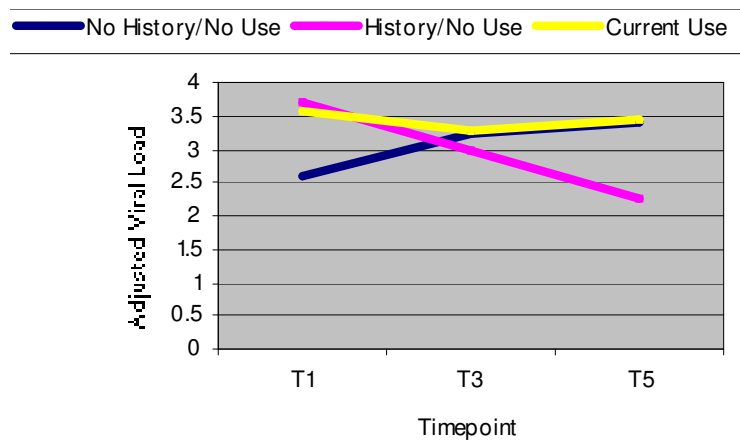

Fig. 5: Changes in viral load among low adherers over time

Depression: There were no significant differences in overall depression $(F[2,212]=.27, p=.76)$, cognitive depression $(F[2,212]=.16, p=.89)$, or somatic depression $(F[2,212]=.40, p=.67)$ at follow-up. Additionally, there were no changes in overall, cognitive, or somatic depression over time for never users, former users, or current users.

Beliefs and concerns: There were no significant differences among the three groups of women on these issues.

Coping/social support: Among the three groups, former users maintained higher levels of social support than both never users $(t[176]=-3.55, p=.001)$ and current users $(t[116]=2.02, p=.045)$. No differences were found in coping or ESS between never users and current users.

Self-efficacy: Overall, there was a significant increase in self-efficacy at long-term follow-up $(t(227)=-2.54$, $p=.012)$. Among the three groups, there were no significant differences at follow-up, neither between nor within groups.

Viral Load: There was a significant decrease in $\log$ viral load for former users post-intervention $(t[1,6]=$ $3.441, p=.01)$, as well as a significant increase in viral load for never users $(t[1,12]=-2.521, p=.03)$. Results are presented in Fig. 5. 
Am. J. Infect. Dis., 3 (4): 240-247, 2007

\section{DISCUSSION}

We examined the role of substance use on medication adherence among HIV seropositive women receiving antiretroviral therapy. Most women reported high levels of adherence at study entry. At baseline, there did not appear to be any difference in medical practitioners' prescribing of medications between those women who are currently substance users, former users and those who reported no history of substance abuse. In addition, current users, former users and never users reported similar levels of adherence to ARVs. Thus, this study supports the supposition that physicians are not discriminating against those who are current substance users in their prescription of ARVs to HIV seropositive women. The one exception may be injecting drug users (IDUs); none of the 338 participants receiving ARVs self-identified as being an IDU.

With the exception of current crack cocaine users, a significant proportion of current users who reported adherence rates of $90 \%$ or higher had undetectable levels of viral load. Previous studies found that substance use negatively influenced adherence, which subsequently had an impact on viral load (Arnsten, Demas, Grant, Gourevitch, Farzadegan, Howard et al., 2002). While participants in general appeared to overreport their adherence rates, the objective data obtained via viral load corroborates that women who are current substance users (with the exception of crack cocaine users) were capable of managing their regimens (Fig. 2 ). Future studies might utilize additional objective methods of monitoring adherence ${ }^{[25]}$ to increase the overall accuracy of these reports.

Results suggest that certain types of medication are associated with higher adherence rates than others. Among this sample, NRTIs prescription was associated with greater medication compliance than NNRTIs, PIs and other combination treatments. It is likely that the greater numbers of medications and those medications associated with greater numbers of side effects (e.g., PIs) may decrease adherence, in contrast to those women on fewer doses per day or fewer pills per dose. However, this finding was noted only for never users; adherence rates remained unaffected by medication type for both current and former users

Former users reported high levels of participation in groups such as Narcotics Anonymous (NA) and Alcoholics Anonymous (AA). Membership in NA and AA would imply that such participation was associated with increased levels of social support; lower levels of social support have been related to decreased adherence $^{[26,27]}$ and lack of support for medication adherence may discourage individuals from taking their medications ${ }^{[28]}$. Further, these support groups have demonstrated increases in self-efficacy ${ }^{[29]}$, which has been associated with increased adherence ${ }^{[30]}$. Consistent with these findings, our results indicate that these former users report high levels of both social support and self-efficacy.

Depression among former users was more prominent at baseline than among current and never users, which may be attributed to another aspect of recovery programs, which include the acceptance of personal responsibility for one's behavior during periods of substance abuse and taking action to redress the wrongs committed against self and others. Long term follow-up, however, did not reveal associations between adherence and depression in any of the three groups of "low adherers".

Former users who initially identified as "low adherers" showed the largest increase in level of adherence as well as significant reductions in viral load by the conclusion of the study. These participants may have benefited the most from the intervention, which focused on recognizing "triggers" for risk behaviors related both to sexual health and medication adherence. The social support gained from the intervention ${ }^{[31]}$ may be comparable to social support gained in treatment facilities and organizations such as Alcoholics Anonymous or Narcotics Anonymous and thus, these individuals may have been more familiar with and responsive to the methods used in the intervention. As noted above, this finding was not mediated by changes in depression scores, suggesting that improved adherence among former users was a direct effect of the intervention itself.

Most participants maintained positive beliefs regarding the importance of medication, In contrast to previous findings, however, ${ }^{[28,32-34]}$ health related beliefs, attitudes and concerns were not related to the level of adherence. This may be a result of the "ceiling effect" with most of our participants considering themselves to be at least $90 \%$ adherent.

There are several limitations to the findings reported in this study. As noted above, relying exclusively on self reported adherence in all likelihood limited the accuracy of adherence evaluation. Other measures of adherence, such as electronic drug monitoring or pill counts would provide additional supportive data. Although self-report measures are useful for assessing non-adherence ${ }^{[1,26]}$, many participants, particularly current substance users, tend to overestimate their adherence level with self-report measures $^{[1,35]}$ or may not consider themselves nonadherent when they modify their own 
regimen ${ }^{[36]}$.Overestimation as a consequence of current substance use may also explain the high levels of selfefficacy endorsed by current users at baseline, comparable to the alcoholic's estimation of their driving skills while "under the influence".

This intervention was successful in increasing adherence among former drug using low adhering women. This provides support for the use of medication adherence interventions utilizing a combination of social support and psychoeducational methods for this population of women living with HIV. Additional research is necessary to identify effective interventions for low adhering current drug users and those with no drug history. However, the study does provide support for the prescription of ARVS to current as well as former substance users. Results also suggest that special attention to current crack cocaine users may be necessary to achieve comparable results with this group.

Finally, the absence of current injecting drug users (IDUs) in our sample suggests bias among physicians against prescribing ARVs to this population. The impact of this bias is seen in Fig. 1 from the parent sample $(n=481)$ which illustrates the significantly higher log viral loads among this group as compared with users of other drugs. The rationale for withholding ARVs from current IDUs is well known (poor adherence leading to increases in drug-resistant strains, etc), but the issue of treatment for these patients remains unresolved. It is clear that this group represents a special challenge to those concerned with medication adherence research and that our efforts to prevent infection and control transmission of HIV must include such underserved segments of our target population.

\section{ACKNOWLEDGEMENTS}

We are pleased to acknowledge support for this study from the National Institute of Mental Health, NIH (R01MH 55463 and R01MH61208). We also would like to acknowledge the contributions of our research teams at the University of Miami Miller School of Medicine, the Clinical Directors Network and St. John's University, the community sites providing referrals and, most importantly, our study participants.

\section{REFERENCES}

1. Paterson, D.L., B., Potoski and B. Capitano, 2002. Measurement of adherence to antiretroviral medications. J. Acquired Immune Def. Syndr., 31 (Suppl.3): S103-S106.
2. Paterson, D.L., S. Swindells, J. Mohr, M. Brester, E.N. Vergis and C. Squier et al., 2000. Adherence to protease inhibitor therapy and outcomes in patients with HIV infection. Ann. Int. Med., 133 (Suppl. 1): 21-30.

3. Deeks, S., G. Beatty, P.T. Cohen, R. Grant and P. Volberding, 1998. Viral load and CD+ T cell changes in patients failing potent protease inhibitor therapy. [abstract] $5^{\text {th }}$ Conference on Retroviruses and Opportunistic Infections, Chicago, IL.

4. Boden, D., A. Hurley, L. Zhang, Y. Cao, Y. Guo and E. Jones et al., 1999. HIV-1 drug resistance in newly infected individuals. J. Am. Med. Assoc., 282: 1135-1141.

5. Carpenter, C.C.J., M.A. Fischl, S.M. Hammer, M.S. Hirsch, D.M. Jacobsen, D.A. Katsenstein and J.S.G. Montaner et al., 2000. Antiretroviral therapy in adults updated recommendations of the international AIDS society-USA panel. J. Am. Med. Assoc., 283: 381-390.

6. Hogg, R.S., B. Yip, K. Chan, M.V. O'Shaughnessy J.S.V. Montaner, 2000. Non-adherence to triple combination is predictive of AIDS progression and death in HIV positive men and women. $7^{\text {th }}$ Conf. Retroviruses and Opportunistic Infections.

7. Reiter, G.S., K.E. Stewart and L. Wojtusik, 2000. Elements of success in HIV clinical care: Multiple interventions that promote adherence. Topics in HIV Medicine, 8 (Suppl.5): 21-30.

8. Mcpherson-Baker, S., R. Malow, F. Penedo, D. Jones, N. Schneiderman and N. Klimas, 2000. Enhancing adherence to combination antiretroviral therapy in non-adherent HIV-positive men. AIDS Care, 12: 399-404.

9. Chander, G., Lau, B., \& Moore, R.D. 2006. Hazardous alcohol use: A risk factor for nonadherence and lack of suppression in HIV infection. J. Acquir. Immune Defic. Syndr., 43: 411- 417.

10. Hinkin, C.H., T.R. Barclay, S.A. Castellon, A.J. Levine, R.S. Durvasula and S.D. Marion et al. 2007. Drug use and medication adherence among HIV-1 infected individuals. AIDS and Behavior, 11: 185-194.

11. Shannon, K., T. Kerr, C. Lai, T. Ishida, E. Wood and J.S. Montaner et al., 2005. Nonadherence to antiretroviral therapy among a community with endemic rates of injection drug use. J. Intl. Assoc. Physicians in AIDS Care, 4: 66 - 72.

12. Liu, H., D. Longshore, J.K. Williams, I. Rivkin, T. Loeb and U.S. Warda, et al., 2006. Substance abuse and medication adherence among HIVpositive women with histories of child sexual abuse. AIDS and Behavior, 10: 279 - 286. 
13. Wong, M.D., W.E. Cunningham, M.F. Shapiro, R.M. Andersen, P.D. Cleary and N. Duan et al., 2004. Disparities in HIV treatment and physician attitudes about delaying protease inhibitors for nonadherent patients. J. Gen. Int. Med., 19: 366-374

14. Segal-Isaacson, C.J., J.N. Tobin, S.M. Weiss, E. Brondolo, A. Vaughn and C. Wang et al. Improving Dietary Habits In Disadvantaged Women With HIV/AIDS: The SMART/EST Women's Project (in press, AIDS \& Behavior)

15. Chesney, M. and J. Ickovics, 1997. For the recruitment, adherence and retention committee of the ACTG: Adherence to combination therapy in AIDS clinical trials. Presented at the Annual Meeting of the AIDS Clinical Trials Group, Washington, D.C.

16. Beck, A.T., R.A. Steer and M.G. Garbin, 1988. Psychometric properties of the BDI: 25 years later. Clin. Psychol. Rev., 8: 77-100.

17. Beck, A.T., C.H. Ward, M. Mendelson and J. Erbaugh, 1961. An inventory for measuring depression. Arch. Gen. Psych., 4: 561-571.

18. Horne, R., J. Weinman and M. Hankins, 1991. The beliefs about medicines questionnaire: The development and evaluation of a new method for assessing the cognitive representation of medication. Psychology and Health, 12: 1-24.

19. Carver, C., M. Sheier and J.D. Weintraub, 1989. Assessing coping strategies: A theoretically based approach. J. Person. Soc. Psychol., 56: 267-283.

20. Bandura, A., 1995. Self-efficacy and changing societies. New York: Cambridge University Press.

21. Creuss, S., M.H. Antoni, A. Hayes, F.J. Penedo, G. Ironson and M.A. Fletcher et al., 2002. Changes in mood and depressive symptoms and related change processes during cognitive behavioral stress management in HIV-infected men. Cogn. Ther. and Res., 26: 373-392.

22. Beck, A.T., J. Rush, B. Shaw and G. Emery, 1979. Cognitive therapy of depression. New York: Guilford.

23. Barre-Sinoussi, F., J.C. Chermann, F. Rey, M.T. Nugeyre, S. Chamaret and J. Gruest, 1983. Isolation of a T-lymphotropic retrovirus from a patient at risk for acquired immune deficiency syndrome (AIDS). Science, 220: 868-871.

24. Kievits, T., B. van Gemen, D. van Strijp, R. Schukkink, M. Dircks and H. Adriaanse, 1991. NASBA isothermal enzymatic in vitro nucleic acid amplification optimized for the diagnosis of HIV-1 infection. J. Virol. Methods, 35: 273-286.

25. Arnsten, J.H., P.A. Demas, R.W. Grant, M.N. Gourevitch, H. Farzadegan and A.A. Howard et al., 2002. Impact of active drug use on antiretroviral therapy adherence and viral suppression in HIVinfected drug users. J. Gen. Int. Med., 17: 377-381.
26. Catz, S.L., J.A. Kelly, L.M. Bogart, E.G. Benotsch, and T. Mcauliffe, 2000. Patterns, correlates and barriers to medication adherence among persons prescribed new treatments for HIV disease. Health Psychol., 19 (Suppl. 2): 124-133.

27. Gordillo, V., J. Del Amo, V. Soriano and J. Gonzlez-Lahoz, 1999. Sociodemographic and psychological variables influencing adherence to antiretroviral therapy. AIDS, 13 (Suppl. 13): 17631769.

28. Remien, R.H., A.E. Hirky, M.O. Johnson, L.S. Weinhardt, D. Whittier and G. Minh Le, 2003. Adherence to medication treatment: A qualitative study of facilitators and barriers among a diverse sample of HIV+ men and women in four U.S. cities. AIDS and Behavior, 7 (Suppl. 1): 61-72.

29. Bogenschutz, M.P., J.S. Tonigan and W.R. Miller, 2006. Examining the effects of alcoholism typology and AA attendance on self-efficacy as a mechanism of change. J. Studies on Alcohol, 67: $562-567$.

30. Barclay, T.R., C.H. Hinkin, S.A. Castellon, K.I. Mason, M.J. Reinhard and S.D. Marion et al., 2007. Age-associated predictors of medication adherence in HIV-positive adults: health beliefs, self-efficacy and neurocognitive status. Health Psychol., 26, 40 - 49.

31. Jones, D.L., S. MacPherson-Baker, D. Lydston, Y. Gousse, E. Brondolo, J. Camille, J.N. Tobin and S.M. Weiss, 2007. Influencing medication adherence among HIV+ women: The SMART/EST II Women's Project. AIDS \& Behavior, 11: 79-86.

32. Wagner, G.J., R.H. Remien, A. Carballo-Dieguez, and C. Dolezal, 2002. Correlates of adherence to combination antiretroviral therapy among members of HIV-positive mixed status couples. AIDS CARE, 14 (Suppl.1): 105-109.

33. Malcolm, S.E., J.J. Ng, R.K. Rosen and V.E. Stone, 2003. An examination of HIV/AIDS patients who have excellent adherence to HAART. AIDS Care, 15 (Suppl. 2): 251-261.

34. Sankar, A., M. Luborsky, P. Schuman and G. Roberts, 2002. Adherence discourse among African-American women taking HAART. AIDS Care, 14 (Suppl.2): 203-218.

35. Bangsberg, D.R., F.M. Hecht, H. Clague, E.D. Chalebois, D. Ciccarone and M. Chesney et al., 2001. Provider Assessment of Adherence to HIV Antiretroviral Therapy. J. Acquir. Immune Defic. Syndr., 26 (suppl. 5): 435-442.

36. Hill, Z., C. Kendall and M. Fernandez, 2003. Patterns of Adherence to Antiretrovirals: Why Adherence Has No Simple Measure. AIDS Patient Care STDs, 17 (Suppl 10): 519-525. 